\section{Baculovirus expression system for magnetic sorting of infected cells and enhanced titer determination}

\author{
Björn Philipps, Michael Forstner, and Lorenz M. Mayr \\ Novartis Pharma AG, Basel, Switzerland \\ BioTechniques 36:80-83 (January 2004)
}

Recombinant baculoviruses derived from the Autographa californica nuclear polyhedrosis virus (AcNPV) are widely used to express heterologous genes in insect cells, but the use of the baculovirus expression vector system (BEVS) is hampered by slow and tedious procedures for the selection and separation of baculovirus-infected insect cells and for titer determination. Here we developed a new technology based on the bicistronic vector with a fusion protein of the human integral plasma membrane glycoprotein CD4 and green fluorescent protein (GFP) for concomitant expression of target proteins in insect Sf21 cells. Magnetic cell sorting (MACS) technology with anti-CD4 antibody-labeled superparamagnetic beads was used to separate the baculovirus-infected from the noninfected insect cells and therefore to increase the virus titer and to reduce process time. With the herein described use of the MACS-improved baculovirus expression plasmid MACS in baculovirus expression (pMACSiBac-1), we have been able to select the baculovirus-infected insect cells at an early time point of the infection cycle and therefore enrich the virus titer dramatically. Furthermore, simple end point dilution and GFP fluorescence detection can be used for early and facile detection of recombinant viruses and simplified titer determinations. We show that the bicistronic pMACSiBac-1 with an additional multiple cloning site under the control of the very late promoter polyhedrin $\left(P_{P H}\right)$ allows for the expression of target proteins in high amounts, less workloads, and shorter timelines.

\section{INTRODUCTION}

Recombinant baculoviruses are widely used to express heterologous genes in insect cells (1). The baculovirus expression vector system (BEVS) has many advantages, such as the capacity for large DNA insertion and a high yield of recombinant protein. The similarity of the proteins produced in BEVS to the naturally occurring human proteins in terms of posttranslational modifications (e.g., phosphorylation), biological activity, and protein stability is one reason for the widespread use of BEVS in academia and industry (1). Despite these advantages, the use of BEVS is hampered by slow and tedious procedures for the selection and separation of baculovirus-infected insect cells and for virus titer determination. Here we report a technology to successfully address the two main bottlenecks for protein production in insect cells by a combined approach using green fluorescent protein (GFP) fluorescence and magnetic cell sorting
(MACS) technology.

GFP from the jellyfish Aequorea victoria has become an excellent marker, not only for studies on gene expression, protein localization, and dynamics in various biological systems, but also for protein purification and protein interaction studies (2-4). The use of GFP in a baculovirus expression system allows for the early and facile detection of recombinant viruses and simplifies titer determinations (5).

The separation of $\mathrm{T}$ lymphocytes from other human cells is a technique often used in clinical analytics (6). It is mainly performed with labeled antibodies against a cell type-specific membrane protein (e.g., CD4, CD8, CD34). The antibodies are either labeled with a fluorescent dye and the separation is performed via fluorescence-activated cell sorting (FACS) or the antibody is labeled with superparamagnetic beads and MACS is used for separation. The advantage of the MACS technique over FACS is a much higher throughput. Approximately $10^{9}-10^{10}$ cells can be sorted by MACS in about $1 \mathrm{~h}$, whereas FACS is only able to sort $1 \%$ of that amount in the same time $(7,8)$.

In this study, we used the MACS technology with anti-CD4 MicroBeads $^{\mathrm{TM}}$ to select the baculovirus-infected insect cells, expressing the integral plasma membrane glycoprotein CD4. In the commercially available bicistronic vectors (e.g., pFastBac ${ }^{\mathrm{TM}}$ DUAL), gene expression is driven by two very late promoters, namely polyhedrin $\left(\mathrm{P}_{\mathrm{PH}}\right)$ and p10 promoter. For efficient separation of infected from noninfected cells, a selection at an earlier time point after infection is absolutely necessary to obtain a maximum number of virus-loaded cells. We therefore changed the promoter for expression of the CD4 gene from $\mathrm{p} 10$ to the earlier expressing basic promoter $\left(\mathrm{P}_{\text {Basic }}\right)$. The second multiple cloning site (MCS) after the very late promoter $\mathrm{P}_{\mathrm{PH}}$ is reserved for expression of the target gene. A fusion protein of fulllength CD4 and enhanced GFP (EGFP) was designed to combine the sorting of the infected cells by MACS with the titer determination with EGFP.

\section{MATERIALS AND METHODS}

\section{Plasmid Constructions}

For separation of the infected cells by the MACS technology and titer determination, we designed a fusion construct of full-length CD4 and EGFP linked by a single serine. For construction of this fusion gene, assembled "sticky-end" PCR products (9) with the plasmids $\mathrm{pLNCX}$-hCD4-EGFP-KKXX (10) and pIRES2-EGFP (BD Bioscience, Palo Alto, CA, USA) as templates were ligated into the vector $\mathrm{pFastBacD}$ UAL (Invitrogen, Carlsbad, CA, USA) yielding the plasmid pFBD-CD4eGFP. Two PCR steps were performed as well to exchange the very late $\mathrm{p} 10$ promoter with the late $\mathrm{P}_{\mathrm{Basic}}$ promoter: $(i)$ the promoters $\mathrm{P}_{\mathrm{Basic}}$ and $\mathrm{P}_{\mathrm{PH}}$ were amplified individually from the vectors $\mathrm{pAc}$ MP3 (BD Bioscience Pharmingen, San Diego, CA, USA) and pFastBacDUAL, and then (ii) an assembled PCR product was cloned into pFastBacDUAL yielding the plasmid pFBD-PB. The plasmid pMACSiBac-1 (Figure 1) 


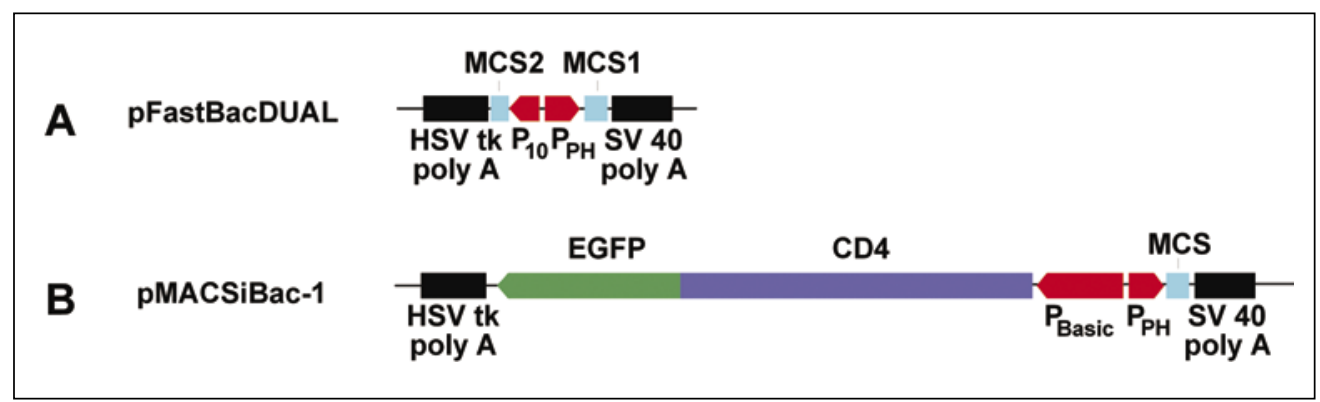

Figure 1. Representation of the relevant portion from the vectors (A) pFastBacDUAL and (B) pMACSiBac1. CD4-EGFP, fusion protein of CD4 (cluster of differentiation 4) and EGFP (enhanced green fluorescent protein); MCS1/2, multiple cloning sites 1/2; MCS, multiple cloning site related to MCS1 and containing the unique restriction sites NcoI, BamHI, RsrII, BssHII, EcoRI, SalI, SpeI, NotI, BstBI, XbaI, PstI, and HindIII; $\mathrm{P}_{10}$, p10 promoter; $\mathrm{P}_{\text {Basic' }}$ basic promoter; $\mathrm{P}_{\mathrm{PH}}$, polyhedrin promoter; HSV tk poly $(\mathrm{A})$, herpes simplex virus thymidine kinase polyadenylation signal; SV40 poly(A), simian virus 40 late polyadenylation signal. was constructed by cloning the CD4EGFP gene from $p F B D-C D 4 e G F P$ into the vector $\mathrm{pFBD}-\mathrm{PB}$. More detailed information about the cloning of the plasmid is provided as supplementary material on the BioTechniques Web site at http://www.BioTechniques.com/ Jan04/PhilippsSupplementary.html. The plasmids are available from the authors upon request.

\section{Virus Production}

Competent Escherichia coli cells of strain DH10Bac ${ }^{\mathrm{TM}}$ (Invitrogen) were transformed with the recombinant plasmid pMACSiBac-1, and colonies containing the recombinant bacmid were grown according to the Bac-to-
(GenBank ${ }^{\circledR}$ accession no. AY438650)
$\mathrm{Bac}^{\mathrm{TM}}$ baculovirus expression system instructions (Invitrogen) for isolation of the resulting bacmid DNA with the help of the Large-Construct DNA-isolation kit (Qiagen, Hilden, Germany). Transfection of approximately $2 \times 10^{8}$ Spodoptera frugiperda (Sf21) cells (Invitrogen) was performed using $140 \mu \mathrm{L}$ Cellfectin $^{\mathrm{TM}}$ reagent (Invitrogen) and $20 \mu \mathrm{g}$ bacmid DNA. At $48 \mathrm{~h}$ after transfection, Sf21 cells were labeled with anti-CD4 MACS MicroBeads, and the separation was performed on an autoMACS ${ }^{\text {TM }}$ system according to the user manual (both from Miltenyi Biotec, Bergisch Gladbach, Germany). The cells were seeded, and fluorescence and phase contrast pictures were taken $2 \mathrm{~h}$ after sorting (Figure 2), and the virus was harvested $48 \mathrm{~h}$ after sorting.

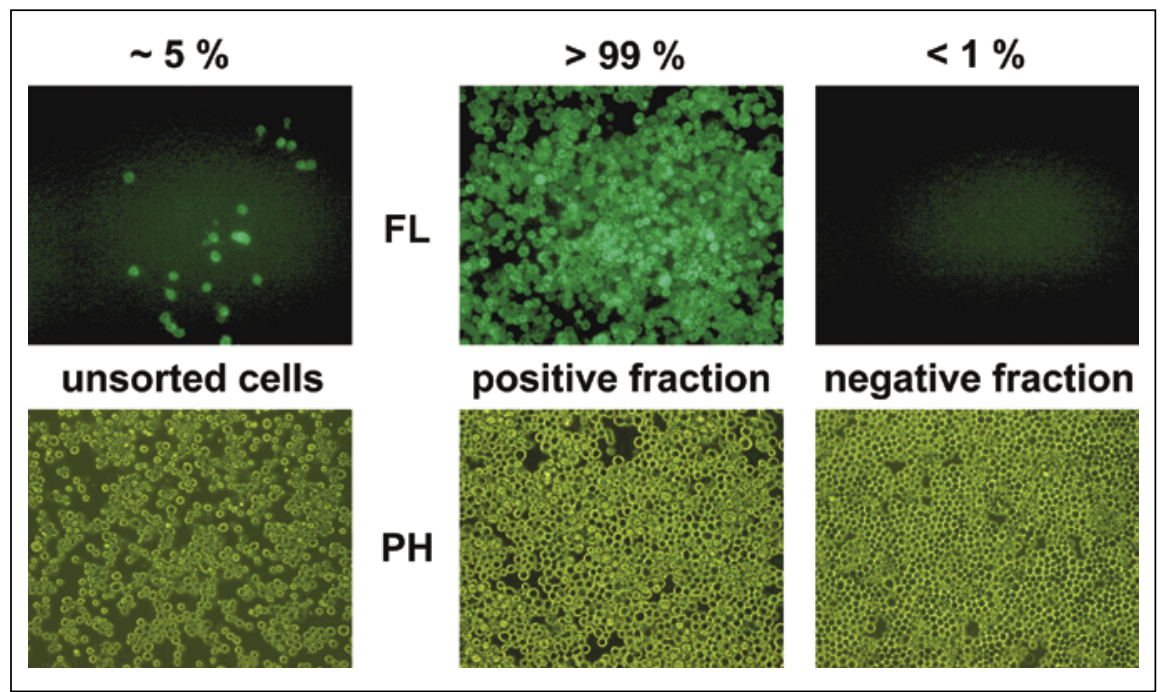

Figure 2. Fluorescence (top) and light (bottom) micrographs of bMACSiBac-1-transfected Sf21 cells $2 \mathrm{~h}$ after sorting on an autoMACS system. FL, fluorescence; PH, phase contrast.

\section{Titer Determination}

The titer of the generated high titer virus stock was determined with end point dilution and GFP fluorescence detection. Therefore, $10-\mu \mathrm{L}$ aliquots of serial dilutions of the high titer stock virus $\left(10^{-2}\right.$ to $10^{-9}$ dilution) were mixed with $100-\mu \mathrm{L}$ aliquots of $\mathrm{Sf} 21$ cell suspension $\left(10^{5}\right.$ cells/ $\mathrm{mL}$ ), and 12 aliquots of each mixture were seeded into a 96well plate (5). After 2 days, the number of wells in which GFP fluorescence was detected with at least one cell was counted (Figure 3). The virus titer was calculated according to the $50 \%$ tissue culture infectious dose $\left(\mathrm{TCID}_{50}\right)$ method (11). For a detailed description and statistical basis of the method please see References 1, 12, and 13. The titer determination with the BacPAK ${ }^{\mathrm{TM}}$ Rapid Titer kit was done according to the user manual (BD Bioscience).

\section{Expression Analysis of $\beta$-Galactosidase as an Example Protein}

Two very similar recombinant bacmids were constructed to analyze the effect of the expression of CD4-EGFP on the expression of a targeted gene. In the first construct, the lac Z gene was cloned into the MCS of pMACSiBac-1, and in the second construct the lac $Z$ gene was cloned into the MCS after the $\mathrm{P}_{\mathrm{PH}}$ promoter of pFBD-PB. Sf21 cells were infected with the two different recombinant baculoviruses with a multiplicity of infection (MOI) of 1 , and cells were harvested 2 days postinfection (p.i.). $\beta$ Galactosidase expression of the samples was analyzed by Western blot analysis with an anti- $\beta$-galactosidase antibody (Sigma-Aldrich, Buchs, Switzerland) and activity measurements with a $\beta-G a l$ Assay kit (Invitrogen) and compared to noninfected insect cells (Figure 4).

\section{RESULTS AND DISCUSSION}

To combine the separation of baculovirus-infected insect cells from the noninfected cells via MACS with a 
fluorescence-based titer determination, we constructed the plasmid pMACSiBac-1. The plasmid was derived from the pFastBacDUAL by exchange of the very late $\mathrm{p} 10$ promoter to the late $\mathrm{P}_{\mathrm{Basic}}$ promoter and cloning of a fusion protein consisting of CD4 and EGFP in the MCS2 after the $\mathrm{P}_{\text {Basic }}$ (Figure 1).

For sufficient selection of infected in-

sect cells, the promoter expressing CD4 was exchanged from the p10 promoter to $\mathrm{P}_{\text {Basic }}$ promoter, which allows the selection of baculovirus-infected cells at an earlier time point p.i. Therefore, the main part of the budded virus particles is not released from the infected cells before selection and can be obtained by harvesting of the cells. The selection

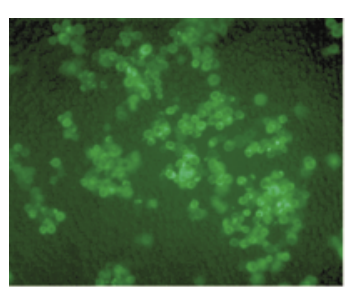

$10 \mathrm{~nL}$

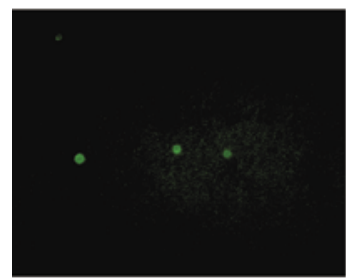

$10 \mathrm{pL}$

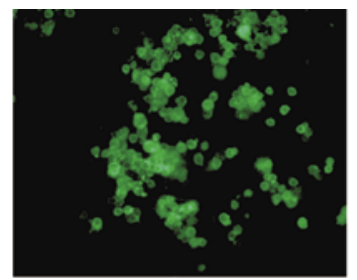

$1 \mathrm{~nL}$

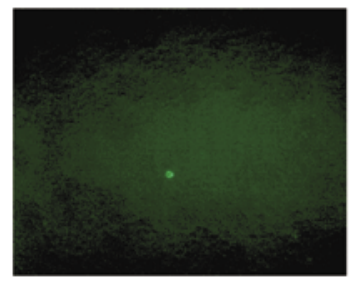

$1 \mathrm{pL}$

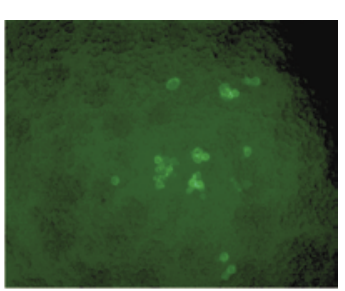

$100 \mathrm{pL}$

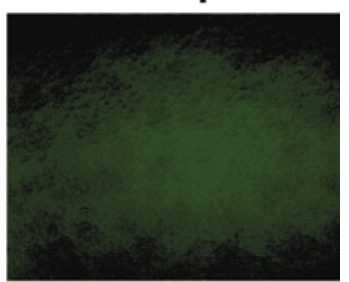

$100 \mathrm{fL}$

Figure 3. Titer determination using green fluorescent protein (GFP). The high titer stock was serially diluted, and $10 \mu \mathrm{L}$ of the different dilutions were added into wells of a 96-well plate and seeded with $100 \mu \mathrm{L}$ Sf 21 insect cells $\left(1 \times 10^{5}\right.$ cells $\left./ \mathrm{mL}\right)$. The fluorescence of the cells was determined 2 days postinfection (p.i.).

\section{A}

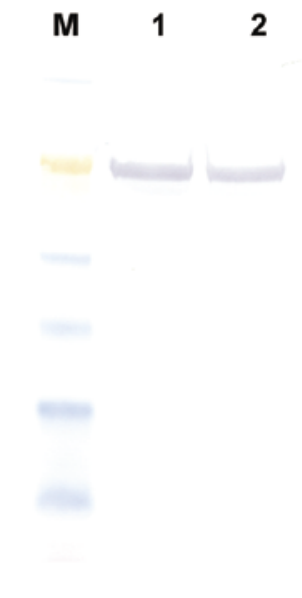

B

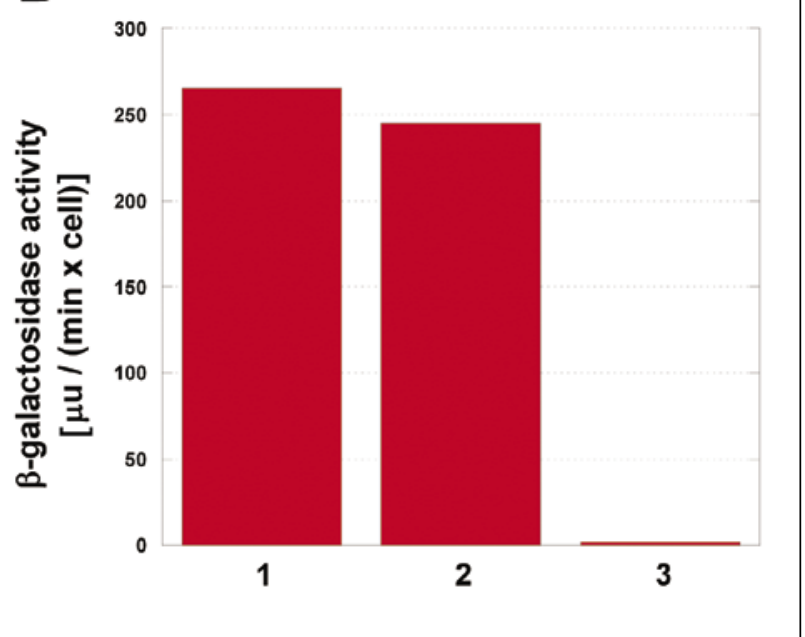

Figure 4. Comparison of the expression yield of a target protein with and without co-expression of the fusion protein CD4-EGFP. (A) Western blot analysis of $\beta$-galactosidase of Sf 21 soluble cell extract 2 days postinfection (p.i.). $\mathrm{M}$, SeeBlue ${ }^{\circledR}$ Plus 2 prestained molecular weight standard (Invitrogen); lane 1, expression of $\beta$-galactosidase and CD4EGFP; lane 2, expression of $\beta$-galactosidase without CD4-EGFP; lane 3, noninfected cells as control (noninfected cells are 100 -fold more concentrated than infected cells). (B) Relative $\beta$-galactosidase activity of the same samples. Numbering as in panel A. $\beta$-Galactosidase activity $(\mathrm{u})$ is defined as amount of hydrolyzed orthonitrophenyl- $\beta$-D-galactopyranoside (ONPG) in nmol according to the $\beta$-Gal Assay kit manual. method was stringent, because at least 99\% of the cells from the positive fraction showed GFP fluorescence, while in the negative fraction, no fluorescent cells could be observed (Figure 2). Using the protocol for the Bac-to-Bac system, two additional virus amplification steps, each taking about 3 days time, are needed. We have been able to speed up the generation of the virus stock by at least 6 days. This optimized baculovirus production leads to a much higher throughput either on produced proteins or on tested constructs of the same number of proteins, which will result in easier protein purification and higher yields of pure proteins.

The effect of the expression of the CD4-EGFP fusion protein of the expression yield of a targeted protein was determined. Therefore the lac Z gene was cloned after the very late promoter $\mathrm{P}_{\mathrm{PH}}$ in the pMACSiBac as well as in the pFBD-PB, and the expression yield of $\beta$-galactosidase was analyzed. Both Western blot and activity analyses showed comparable absolute $\beta$-galactosidase expression for both constructs, whereas the noninfected cells showed no $\beta$-galactosidase activity (Figure 4 ). We conclude therefore, that the expression of the fusion construct CD4-EGFP under the late promoter $\mathrm{P}_{\text {Basic }}$ has no effect on the expression amount of the target gene.

Virus titer of the cells after MACS sorting was determined at $3.5 \times 10^{7}$ plaque-forming units $(\mathrm{pfu}) / \mathrm{mL}$, whereas the unsorted cells showed a titer of $1.7 \times 10^{6} \mathrm{pfu} / \mathrm{mL} \quad(20 \times$ enrichment factor). Titer determination with GFP expressed under the very late promoter $\mathrm{P}_{\mathrm{PH}}$ was performed after 4 days (5), whereas the use of the late promoter $\mathrm{P}_{\mathrm{Basic}}$ for expression of the GFP gene allowed for the titer to be determined 2 days p.i. (Figure 3). Titer determination with the BacPAK Rapid Titer kit also only takes 2 days, but is significantly more work-intensive than the titer determination method described herein. BacPAK 
titer determination for the virus titer stock after sorting shows a titer of $7 \times 10^{7} \mathrm{pfu} /$ $\mathrm{mL}$, which is comparable to the titer determined with the GFP method. With the aid of this new GFP-based titer determination, we have been able to get results comparable to the BacPAK system in the same time but with a significantly reduced workload.

In summary, the vector described herein can be used to generate a high titer stock of baculovirus in a much shorter time compared to the classical protocol of the commercially available and widely used Bac-to-Bac system with the same benefits of the system. This selection method is therefore able to solve the first major bottleneck for protein production by BEVS in insect cells. Moreover, the simultaneous integration of GFP into the bicistronic vector pMACSiBac-1 for baculovirus-based expression in insect cells has two main advantages: $(i)$ the infection cycle of the insect cells can easily be monitored by the fluorescence of the cells, and therefore a more informed decision for the time of cell harvesting can be made, and (ii) the determination of the virus titer utilizing GFP fluorescence is a much faster and easier way than the plaque assay method. Using GFP expression under the late promoter $\mathrm{P}_{\text {Basic }}$ for virus titer determination, we reduced the incubation times by 3 days, from 5 days (14) to 2 days. With the new tools for production and determination of high titer baculovirus stock described, the number of different protein constructs tested at the same time was significantly increased, resulting in a higher number of recombinant proteins, which could be produced with a higher purity. By combining these two techniques, we have been able to reduce the time typically needed for protein production in insect cells dramatically and reach a time frame that is comparable to that of protein production in prokaryotic cells.

\section{ACKNOWLEDGMENTS}

We would like to thank Mr. Micha Wicki for performing the virus titer determination with the BacPAK rapid titer kit and Dr. Blanche Schwappach (ZMBH, Heidelberg, Germany) for providing us with the plasmid pLNCXhCD4-EGFP-KKXX.

\section{REFERENCES}

1.O'Reilly, D.R., L.K. Miller, and V.A. Luckow. 1994. Baculovirus Expression Vectors. Oxford University Press, New York.

2.van Roessel, P. and A.H. Brand. 2002. Imaging into the future: visualizing gene expression and protein interactions with fluorescent proteins. Nat. Cell Biol. 4:E15-E20.

3.Lippincott-Schwartz, J., E. Snapp, and A. Kenworthy. 2001. Studying protein dynamics in living cells. Nat. Rev. Mol. Cell Biol. 2: 444-456.

4.Katagiri, Y. and K.C. Ingham. 2002. Enhanced production of green fluorescent fusion proteins in a baculovirus expression system by addition of secretion signal. BioTechniques 33: 24-26.

5.Cha, H.J., T. Gotoh, and W.E. Bentley. 1997. Simplification of titer determination for recombinant baculovirus by green fluorescent protein marker. BioTechniques 23:782-786.

6.Kantor, A.B., I. Gibbons, S. Miltenyi, and J. Schmitz. 1998. Magnetic cell sorting with colloidal superparamagnetic particles, p. 153-173. In D. Recktenwald and A. Radbruch (Eds.), Cell Separation Methods and Applications. Marcel Dekker, New York.

7.Chalmers, J.J., M. Zborowski, L. Sun, and L. Moore. 1998. Flow through, immunomagnetic cell separation. Biotechnol. Prog. 14:141-148.

8.Miltenyi, S., W. Muller, W. Weichel, and A. Radbruch. 1990. High gradient magnetic cell separation with MACS. Cytometry 11: 231-238.

9.Pham, K., K.S. LaForge, and M.J. Kreek. 1998. Comparison of methods for quantitation of radioactivity in protected hybrids in RNase protection assays. BioTechniques 25:198-204.

10.Zerangue, N., M.J. Malan, S.R. Fried, P.F. Dazin, Y.N. Jan, L.Y. Jan, and B. Schwappach. 2001. Analysis of endoplasmic reticulum trafficking signals by combinatorial screening in mammalian cells. Proc. Natl. Acad. Sci. USA 98:2431-2436.

11.Reed, L.J. and H. Muench. 1938. A simple method of estimating fifty per cent endpoints. Am. J. Hyg. 27:493-497.

12.Hughes, P.R. and H.A. Wood. 1986. In vivo and in vitro bioassay methods for baculoviruses, p. 1-30. In R.R. Granados and B.A. Federici (Eds.), The Biology of Baculoviruses. CRC Press, Boca Raton.

13.Dougherty, R.M. 1964. Animal virus titration techniques, p. 169-223. In R. Harris (Ed.), Techniques in Experimental Virology. Academic Press, New York.

14.Lee, H.H. and L.K. Miller. 1978. Isolation of genotypic variants of Autographa californica nuclear polyhedrosis virus. J. Virol. 27: 754-767.

Received 30 July 2003; accepted 16 October 2003.

Address correspondence to Björn Philipps, Novartis Pharma AG, Lead Discovery Center (LDC), CH-4002 Basel, Switzerland. e-mail: Bjoern.Philipps@pharma.novartis.com 